\title{
Stress inducible proteinase inhibitor diversity in Capsicum annuum
}

\author{
Manasi Mishra', Neha Mahajan ${ }^{1}$, Vaijayanti A Tamhane ${ }^{1,3}$, Mahesh J Kulkarni ${ }^{1}$, lan T Baldwin², Vidya S Gupta ${ }^{1}$ \\ and Ashok P Giri ${ }^{*}$
}

\begin{abstract}
Background: Wound-inducible Pin-II Proteinase inhibitors (PIs) are one of the important plant serine Pls which have been studied extensively for their structural and functional diversity and relevance in plant defense against insect pests. To explore the functional specialization of an array of Capsicum annuum (L.) proteinase inhibitor (CanPls) genes, we studied their expression, processing and tissue-specific distribution under steady-state and induced conditions. Inductions were performed by subjecting $C$. annuum leaves to various treatments, namely aphid infestation or mechanical wounding followed by treatment with either oral secretion (OS) of Helicoverpa armigera or water.
\end{abstract}

Results: The elicitation treatments regulated the accumulation of CanPls corresponding to 4-, 3-, and 2-inhibitory repeat domains (IRDs). Fourty seven different CanPl genes composed of 28 unique IRDs were identified in total along with those reported earlier. The CanPl gene pool either from uninduced or induced leaves was dominated by 3-IRD Pls and trypsin inhibitory domains. Also a major contribution by 4-IRD CanPI genes possessing trypsin and chymotrypsin inhibitor domains was specifically revealed in wounded leaves treated with OS. Wounding displayed the highest number of unique CanPIs while wounding with OS treatment resulted in the high accumulation of specifically CanPl-4, -7 and -10 . Characterization of the PI protein activity through two dimensional gel electrophoresis revealed tissue and induction specific patterns. Consistent with transcript abundance, wound plus OS or water treated C. annuum leaves exhibited significantly higher PI activity and isoform diversity contributed by 3- and 4-IRD CanPls. CanPl accumulation and activity was weakly elicited by aphid infestation yet resulted in the higher expression of CanPl-26, -41 and -43 .

Conclusions: Plants can differentially perceive various kinds of insect attacks and respond appropriately through activating plant defenses including regulation of PIs at transcriptional and post-translational levels. Based on the differentially elicited CanPl accumulation patterns, it is intriguing to speculate that generating sequence diversity in the form of multi-IRD PIs is a part of elaborative plant defense strategy to obtain a diverse pool of functional units to confine insect attack.

Keywords: Plant-insect interaction, Herbivory, Oral secretions, Pin-Il type proteinase inhibitors, CanPI

\section{Background}

Plants have evolved elaborate defense strategies composed of constitutive and inducible responses in order to cope with herbivore challenges. The induced defenses commence only when herbivore-derived signals are perceived by the plants. A wide array of studies has reported the induction of direct and indirect plant defenses in response

\footnotetext{
*Correspondence: ap.giri@ncl.res.in

'Plant Molecular Biology Unit, Division of Biochemical Sciences, CSIR-National Chemical Laboratory, Dr. Homi Bhabha Road, Pune, MS 411 008, India Full list of author information is available at the end of the article
}

to herbivory and other biotic stresses [1-4]. Insect damage, mechanical wounding and/or elicitors in insect oral secretions (OS), such as fatty acid amino acid conjugates, volicitin, inceptins, caeliferins, and glucose oxidase, stimulate the local and systemic release of signaling intermediates like systemin and/or jasmonic acid; these then amplify the defense cascade throughout the plant [5-7]. Though the major consequence of herbivory is wounding, plants' responses to insect feeding are more complex due to the elicitors present in insect OS [8]. Defense responses entail the regulated activation of plant defense genes and the

\section{Biomed Central}


suppression of growth-related genes [8,9]. As a result, defensive metabolites and/or proteins accumulate in plants within the local tissues damaged by herbivores as well as systemically in undamaged tissues.

The accumulation of trypsin and chymotrypsin-like proteinase inhibitors (PIs) throughout the aerial tissues of tomato and potato plants was demonstrated to be a direct consequence of insect-mediated damage or mechanical wounding [10]. Thus, serine PIs represent one of the best examples of locally and systemically induced responses in Solanaceous plants [11-16]. The constitutive expression of PIs, which has been reported to occur in storage organs and the reproductive tissues of plants, may fulfill anti-insecticidal as well as other endogenous functions in planta [4,13,16-18].

Most Solanaceae members contain the multi-gene family encoding Pin-II type PIs $[4,16,19]$, which possesses considerable sequence diversity resulting from variations in tandem sequence repeats, domain duplications and circularly permuted domain organizations [20]. A distinct feature of these PIs is the presence of tandem repeats of a 50-amino-acid polypeptide called inhibitory repeat domain (IRD), which can vary from 1 to 8 with interconnecting linker peptides. Each IRD contains 8 conserved cysteines (Cys) along with a reactive site for targeting a serine protease. Gene duplication events have resulted in the evolution of the multi-domain Pin-II family of PIs with structurally and functionally divergent IRDs [21]. Horn et al. [22] isolated a set of IRDs resulting from the differential proteolysis at the linker peptide separating the subunits of a 7-domain precursor from methyl-jasmonate-elicited $N$. attenuata leaves. The sequence variability in the multi-gene family of Pin-II PI proteins, their regulated expression and their posttranslational processing are together responsible for generating a PI cocktail effective in defense and/or endogenous function $[4,16,23]$.

Several different PI proteins and genes with 1- to 4-IRDs have been identified and characterized from C. annuum (CanPIs) tissues [16,24-29]. There was substantial variability in the induced expression of CanPIs upon aphid infestation, virus infection, chewing by insects and mechanical wounding. The abundance of transcripts did not always result in higher CanPI proteins, though they were well correlated in lepidopteran-infested $C$. annuum leaves. Furthermore, these studies indicated that many CanPIs are expressed simultaneously, but the significance of such PI expression diversity in C. annuum remains unclear.

In order to examine the potential functional specificities of the various isoforms of CanPI in C. annuum, we addressed the following questions: (i) Does elicitation increase PI isoform diversity? (ii) How specialized is the induction response to a particular treatment? Following experimental inductions of $C$. annuum leaves, we investigated the diversity in CanPI transcript and protein profiles. Sequencing revealed 24 novel CanPI transcripts, increasing the total known to 47. Selective analysis of PI activity in proteomes using $1 \mathrm{D}$ and 2D electrophoresis followed by mass spectrometry revealed local and systemic responses in PI activity.

\section{Results}

\section{Differential regulation of CanPls upon induction}

The amplification of cDNA derived from uninduced and induced leaves with CanPI gene-specific oligonucleotides yielded transcripts of 789, 614, 445 and $267 \mathrm{bp}$, representing 4-, 3-, 2- and 1-IRD CanPIs, respectively. Semi-quantitative analysis revealed differential CanPI expression in uninduced and induced leaves (Figure 1A). In comparison to CanPIs with 2-IRDs, those with 4- and 3-IRDs showed higher abundance in wounded leaves treated with water or OS (Figure 1A). In aphid-infested leaves, while the overall expression of CanPIs was low, 3-IRD transcripts were prominent. The amplified transcripts were cloned and 60 representative clones from each treatment (25 in case of uninduced) were sequenced to confirm the identities of the CanPIs. This analysis detected novel 4-, 3- and 2-IRD subtypes based on variations in amino acid composition. Based on this analysis, in addition to the 23 CanPIs previously reported [16,26,27], 24 novel CanPIs were identified including seven 2-IRD, fourteen 3-IRD, and three 4-IRD CanPIs (Table 1). 1-IRD CanPIs identified in uninduced leaves were not detected under any of the induction treatments. Details regarding the treatmentspecific representation of CanPIs and their IRD composition are summarized in Table 1. The frequency of occurrence of individual CanPIs out of the 60 clones sequenced per treatment was analyzed and is referred to as abundance in leaf under a particular treatment (Table 1).

Variation in the abundance of CanPIs was apparent (Figure 1B), with 3-IRD CanPIs being highest (from 40 to $60 \%$ ) either in uninduced or induced leaves. The abundance of 4-IRD CanPIs was increased in leaves subjected to wounding and treated with OS (38\%), compared to aphid-infested (12\%) leaves or in wounded leaves treated with water (17\%). The proportion of 2-IRD CanPIs ranged from the lowest $(10 \%)$ in wounded leaves treated with OS to the highest (20\%) in aphid infested and wounded leaves treated with water. The differential expression of the various subtypes of CanPIs (with respect to their IRD composition; see Table 1) resulted in an induction-specific CanPI profile. CanPI-4, -7, -10, -24,-41 and -43 showed the highest representation in leaves across all induction treatments (Table 1). In wounded leaves treated with OS, CanPI-4 and -7 showed the highest frequency, whereas 
A

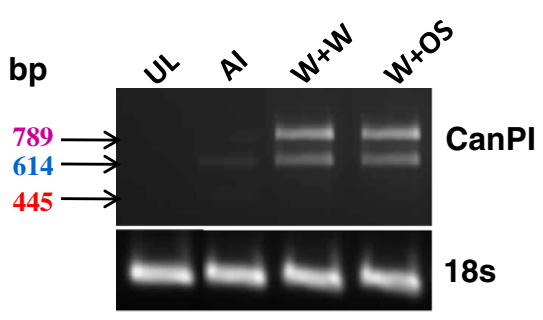

C

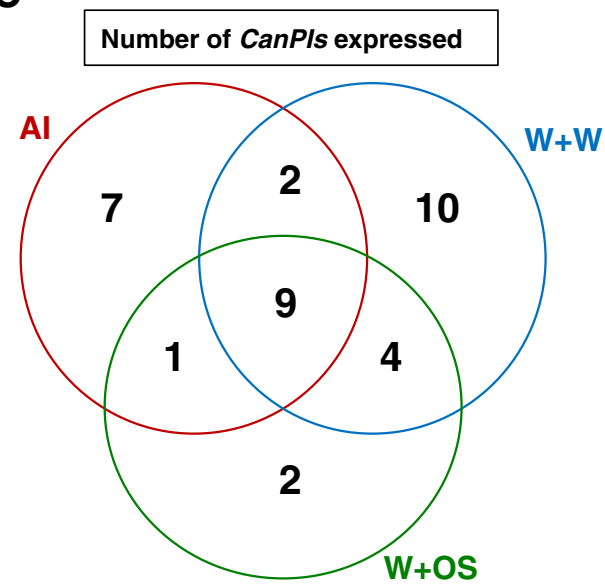

B

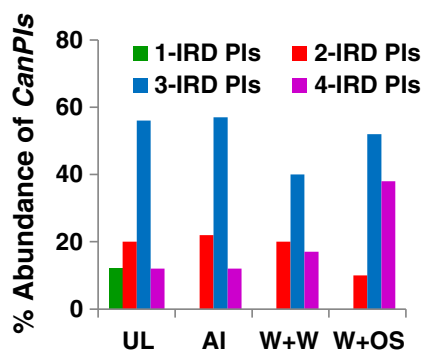

D

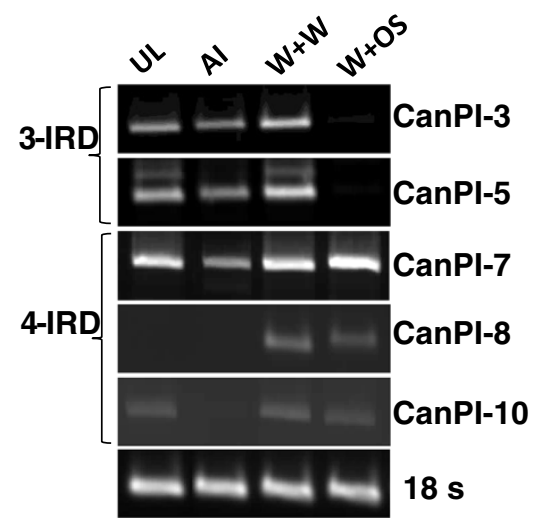

Figure 1 Expression analysis of CanPls under induced conditions. [A] RT-PCR amplification (25 cycles) of mRNA from uninduced (UL) and systemic leaf tissues of induced C. annuum infested with aphids (AI), or wounded and treated with water (W+W) or oral secretions (W + OS). Details of primers used are given in Additional file 1: Table S1. cDNA templates were normalized based on 185 rRNA amplification. [B] Abundance of 1-, 2-, 3- and 4-IRD CanPIs upon each induction treatment to C. annuum leaves. Abundance represents the frequency of a specific type of CanPI in the total clones sequenced per induction. The up-regulation of 4-IRD CanPIs in W+OS is evident. [C] Comparison of CanPl expression patterns in C. annuum leaves after different inductions. The Venn diagram presents the number of CanPls, common or differentially expressed upon each type of induction; Al, W+W or W+OS. W+W showed the highest number of expressed CanPIs. [D] Expression analysis of selected CanPls in un-induced and systemic leaf tissue of induced C. annuum. The details of gene-specific primers are given in Additional file 1: Table S1.

CanPI-41 and -43 were represented most highly in aphid-infested leaves. Wounded leaves treated with water showed the highest number of expressed CanPIs (25) as well as a wide representation of several unique CanPIs (10 in number) in low frequencies (Table 1, Figure 1C). Responses to wounding with OS appear more specialized as suggested by the expression of a few CanPIs (16) in high frequencies (Table 1, Figure 1C). Both the treatments share more CanPIs (3- and 4-IRD type) possibly due to the standardization of the amount of wounding between these two treatments. Aphid-infested leaves also accumulated transcripts of 7 unique CanPIs representing a diverse array of PIs. Only transcripts specific for CanPI-3, -5, -7, -8 and -10 could be analyzed from these tissues. CanPI-7 (4-IRD) showed constitutive expression in uninduced as well as induced leaves, while CanPI-3 and -5 (3-IRD PIs) showed low accumulation levels in wounded leaves treated with OS (Figure 1D). CanPI-8 and -10 (4-IRD PIs) were differentially expressed in wounded leaves treated with water or OS, and completely absent in aphid-infested leaf tissues (Figure 1D).

The Protein Prowler predictor [30] revealed ER signal peptides in all CanPIs. The ER signal peptide (SP) sequences of 25 aa at the N-terminal of CanPIs, which showed ten variants, were named SP-1 to SP-10 (Additional file 2: Figure S1).

To investigate the interrelationships and groupings within CanPIs and with out-groups, phylogenetic analysis was carried out. CanPIs formed a distinct cluster from full-length Pin-II PI (4-IRD) from Nicotiana benthamiana used as an out-group. The dendrogram revealed clustering based on the identical component IRDs in CanPIs (Figure 2). Distinct clusters of 4- and 3IRD PIs were evident with some intermixing for e.g. CanPI-31, -33, -1 and -4. CanPIs showing more aa sequence similarity associated close to each other for e.g. 
Table 1 IRD composition and induction patterns of C. annuum Pin-II PI genes

\begin{tabular}{|c|c|c|c|c|c|c|c|c|c|}
\hline \multirow[t]{2}{*}{ Name } & \multirow[t]{2}{*}{ SP } & \multirow[t]{2}{*}{ 1-IRD } & \multirow[t]{2}{*}{ 2-IRD } & \multirow[t]{2}{*}{ 3-IRD } & \multirow[t]{2}{*}{ 4-IRD } & \multicolumn{4}{|c|}{ Abundance in leaf } \\
\hline & & & & & & $\mathrm{UL}$ & Al & $\mathrm{W}+\mathrm{W}$ & $\mathrm{W}+\mathrm{S}$ \\
\hline CanPl-1 & 5 & 4 & 5 & 10 & & 2 & 1 & & \\
\hline CanPI-2 & 2 & 1 & 16 & 13 & & & & & 1 \\
\hline CanPI-3 & 1 & 1 & 1 & 17 & & 3 & 3 & 2 & 1 \\
\hline CanPI-4 & 1 & 1 & 25 & 17 & & & 3 & 1 & 11 \\
\hline CanPI-5 & 4 & 1 & 1 & 17 & & 6 & 1 & 3 & 3 \\
\hline CanPI-6 & & 2 & 25 & 17 & $p$ & ND & ND & ND & ND \\
\hline CanPI-7 & 5 & 4 & 14 & 5 & 10 & 1 & 5 & 1 & 12 \\
\hline CanPI-8 & 5 & 4 & 14 & 3 & 10 & & & 2 & 3 \\
\hline CanPI-9 & 1 & 1 & 25 & 5 & 10 & ND & ND & ND & ND \\
\hline CanPI-10 & 5 & 4 & 14 & 5 & 8 & 2 & & 3 & 6 \\
\hline CanPI-11 & 1 & 4 & 14 & 5 & 10 & & & 3 & 1 \\
\hline CanPI-12 & & 1 & 1 & 11 & $p$ & ND & ND & ND & ND \\
\hline CanPI-13 & 1 & 17 & & & & 1 & & & \\
\hline CanPI-14 & 1 & 6 & & & & ND & ND & ND & ND \\
\hline CanPI-15 & 5 & 7 & & & & 2 & & & \\
\hline CanPI-16 & 1 & 1 & 17 & & & 1 & & & \\
\hline CanPI-17 & 1 & 12 & 17 & & & 1 & 1 & 1 & 1 \\
\hline CanPI-18 & 1 & 25 & 17 & & & 1 & & & \\
\hline CanPI-19 & 1 & 1 & 25 & & & ND & ND & ND & ND \\
\hline CanPI-20 & 1 & 25 & 17 & & & & & & 3 \\
\hline CanPI-21 & 5 & 4 & 17 & & & 1 & & & \\
\hline CanPI-22 & 5 & 4 & 9 & & & & 1 & 2 & 2 \\
\hline CanPI-23 & 3 & 25 & 17 & & & 1 & & & \\
\hline CanPI-24 & 1 & 1 & 18 & & & & 5 & 4 & \\
\hline CanPI-25 & 3 & 1 & 18 & & & & 2 & & \\
\hline CanPI-26 & 5 & 4 & 10 & & & & 4 & 1 & \\
\hline CanPI-27 & 5 & 23 & 18 & & & & & 1 & \\
\hline CanPI-28 & 8 & 22 & 18 & & & & & 1 & \\
\hline CanPI-29 & 6 & 21 & 18 & & & & & 1 & \\
\hline CanPI-30 & 7 & 1 & 17 & & & & & 1 & \\
\hline CanPI-31 & 1 & 1 & 12 & 27 & 17 & & 1 & & 1 \\
\hline CanPI-32 & 5 & 4 & 14 & 5 & 10 & & 1 & & \\
\hline CanPI-33 & 8 & 24 & 1 & 12 & 17 & & & 1 & \\
\hline CanPI-34 & 1 & 1 & 12 & 17 & & & & 1 & \\
\hline CanPI-35 & 8 & 1 & 12 & 17 & & & & 1 & \\
\hline CanPI-36 & 8 & 5 & 37 & 18 & & & & 1 & \\
\hline CanPI-37 & 8 & 33 & 37 & 48 & & 1 & & 2 & 3 \\
\hline CanPI-38 & 9 & 5 & 40 & 17 & & & & 1 & \\
\hline CanPI-39 & 8 & 5 & 12 & 17 & & & 1 & & \\
\hline CanPI-40 & 5 & 5 & 37 & 18 & & & 1 & & \\
\hline CanPI-41 & 8 & 5 & 37 & 18 & & & 10 & 4 & 5 \\
\hline CanPI-42 & 5 & 4 & 1 & 17 & & & 1 & & \\
\hline
\end{tabular}

Table 1 IRD composition and induction patterns of C. annuum Pin-II PI genes (Continued)

\begin{tabular}{lllllllll}
\hline CanPI-43 & 1 & 1 & 12 & 17 & 2 & 11 & 4 & 4 \\
CanPI-44 & 1 & 1 & 12 & 17 & & & \\
CanPI-45 & 10 & 1 & 12 & 18 & & 2 & \\
CanPI-46 & 1 & 4 & 37 & 18 & 1 & 1 & 1 \\
CanPI-47 & 1 & 1 & 1 & 55 & & 1 & 2 \\
p Partial sequence & & & & & \\
ND Not detected
\end{tabular}

The general architecture of a Pin-II PI gene is displayed on the top of the table. A typical CanPI gene consists of a signal peptide (SP) followed by 1, 2, 3 or 4 IRDs interconnected by linkers. The CanPI genes are listed as follows: 1IRD type; 2-IRD type; 3-IRD type and 4-IRD type. SP denotes signal peptide and the numbers indicate any of the 10 different sequence variants. The position of IRDs is indicated at the top of the columns and the numbers indicate the occurrence of any of the 28 different IRDs at given position. CanPl-1 and -2 are from Kim et al., 2001 and Shin et al., 2001, respectively while CanPl-3 to -23 are from Tamhane et al., 2009. CanPl-24 to -47 were identified in the present work. The frequency of occurrence of individual CanPls in leaves from 60 clones analyzed from each induction treatment is given on the right side of the table. Nucleotide sequences of $\mathrm{CanPl}-24$ to -47 have been submitted to NCBI (Accession numbers JX106474 to JX106497).

CanPI-8, -10 and -11, CanPI-37, -40 and -41, CanPI-13, -16 and -34 .

The deduced amino acid sequences of CanPIs (47 in total) consisted of 28 unique IRDs; of these, 7 had chymotrypsin inhibitory $(\mathrm{CI})$ reactive sites and 21 had trypsin inhibitory (TI) reactive sites. The multiple sequence alignment of IRDs revealed variation mostly within reactive site loops or towards the C-terminal ends (Additional file 3: Figure S2). Five TI IRDs possess 'Lys' while 16 had 'Arg' at the P1 position of the reactive site. CI IRDs had 'Leu' at P1, except IRD 22 which had 'Pro'. IRD 48 (TI) had active site variation with 'Asn' being replaced with 'Asp' (Figure 3A). Another crucial difference was variation in the number and positioning of cysteine residues per IRD. Four such cysteine variants, namely IRD 9, 11 and 24 were TIs, and IRD 33 was a CI (Figure 3A).

The relative abundance of IRDs that resulted from various types of inductions was analyzed (Figure 3B). Representation of IRDs 1, 4, 5, 10, 12, 14, 17, 18, 25 and 37 was high in induced tissues and exhibited treatment specificity. IRDs 1 and 17 were highly represented under all treatments while IRDs 12, 18 and 37, which are TIs, showed high frequency in aphid-infested leaves and wounded leaves treated with water (Figure 3B). The abundance of IRDs 4 and 5, which are CIs, and IRDs 10, 14 and 25, which are TIs, was distinct in wounded leaves treated with OS (Figure 3B). Most of the remaining diversity of IRDs is contributed by wounded leaves treated with water or OS (Figure 3C).

Wounding and insect damage results in quantitative and qualitative changes in CanPI proteins

C. annuum leaves were found to have increased PI activity upon induction (Figure 4A). Significantly higher level 


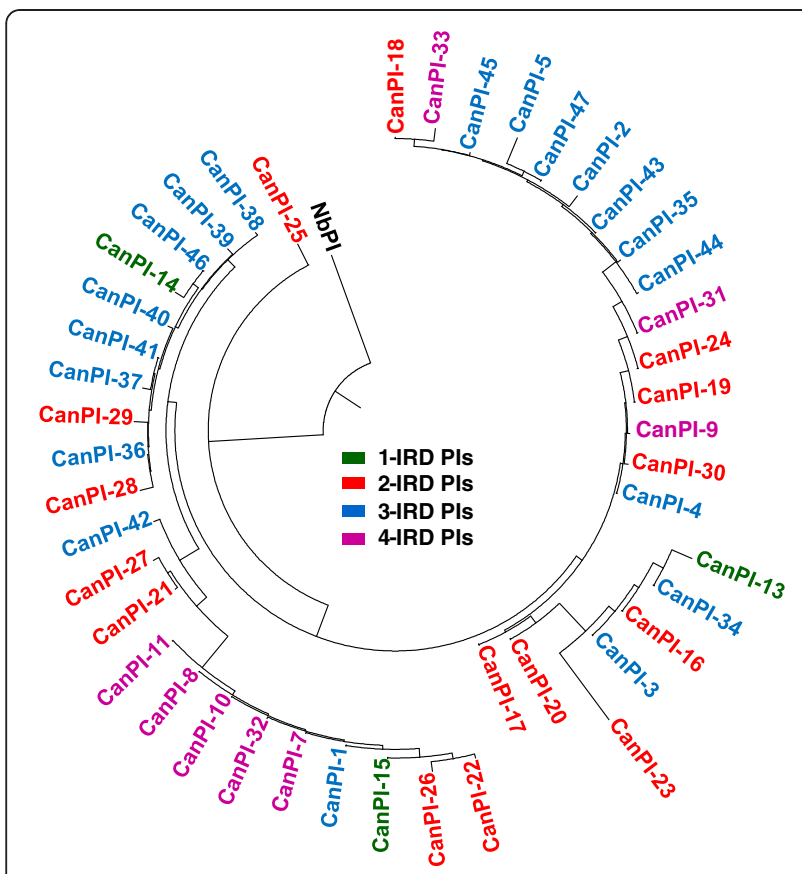

Figure 2 Dendrogram based on aa sequence of CanPIs. Dendrogram of CanPls based on deduced amino acid sequences of full-length genes of 1- to 4-IRDs isolated from C. annuum. Pin-II type PI from N. benthamiana (NbPl, NCBI: ABA42892) is used as an out-group. Details of the CanPls (1 to 47), composition, number of IRDs, tissue-wise representation and color coding are given in Table 1. Dendrogram was constructed using Lasergene software.

of PI activity was evident in the systemic leaves of wounding treated with water induction and both, the local and systemic leaves of wounding treated with OS induction compared to similar leaves from unwounded control plants (Figure 4A). The PI activity in aphidinfested leaves was higher than in uninduced leaves; however, it was 1.5- and 3.5-fold less than in wounded leaves treated with water or with OS, respectively. Induced levels of PI activity ranged from 2-fold in leaves wounded and treated with water to 4-fold in leaves wounded and treated with OS.

A differential pattern of PI isoform induction was observed in C. annuum leaves in response to various treatments (Figure 4B). Three prominent CanPI activity bands were detected in leaves that were wounded and treated with OS or with water, while only two PI isoforms could be detected in case of aphid infested and uninduced leaves. This difference indicated induced qualitative diversity in the CanPIs that resulted from these two treatments. Extracts from leaves wounded and treated with water or OS attained an early saturation of $H$. armigera gut protease (HGP) inhibition (70\%) unlike aphid-infested and uninduced tissues, consistent with the quantitative differences amongst the PIs in their activity (Figure 4C). The HGP of fourth-instar larvae displayed at least seven protease isoforms (HGP-1 to -7), of which only HGP- 6 and -7 were able to retain marginal activity in the presence of CanPIs from either uninduced or induced tissues (Figure 4D).

The 2-D activity profiles of induced leaf samples showed the induction of several novel PI isoforms in the range of isoelectric point (pI) from 4 to 7 and also shift in the $\mathrm{pI}$ and/or molecular mass of few isoforms (Figure 5). Five prominent TI isoforms (TI-1 to TI-5) corresponding to 1-, 2-, 3- and 4-IRD were identified in uninduced leaves, in the pI range of 5 to 7 (Figure 5). TI-6 (1-IRD) isoform which has major basic shift in pI compared to TI-1, was present in all the three types of induced tissues. TI- $3,-4$ and -5 were consistently detected in aphid-infested leaves but were absent from wounded leaves treated with water or OS. TI- 8 to -13 were present only in wounded leaves treated with water, while TI-14, -15, and -16, corresponding to 4-IRD CanPIs, were present in wounded leaves treated with OS only. The induced PI activity in wounded leaves treated with water showed a distribution of several TI isoforms with low intensity, whereas the up-regulation of only a few TI isoforms was evident in wounded leaves treated with OS. TI-2 was absent in all induced tissues, consistent with the specificity of some PI isoforms in uninduced leaves. Among the TI isoforms corresponding to 1-, 2-, 3- or 4-IRD CanPIs, more variations were observed for 3-and 4-IRD CanPIs.

Partially purified PIs contained small peptides of about 5.5 to $6.3 \mathrm{kDa}$ equivalent to a single IRD as analyzed on MALDI-TOF-MS (Figure 6). High molecular mass proteins exhibited very low intensity in the mass spectra, perhaps due to ion suppression effects, and therefore are not considered. Uninduced leaf extracts displayed a single major peak of $5583 \mathrm{Da}$, whereas aphid-infested leaves had major peaks at $5583 \mathrm{Da}$ and $5616 \mathrm{Da}$ and few low intensity peaks. A peak of 5583 Da was most prominent in wounded leaves treated with water, in addition to several peaks of 5616, 5760 and 5832 Da with high intensity. Extract from leaves wounded and treated with OS displayed 6138 and $5961 \mathrm{Da}$ as the major peaks, with low intensity peaks at 5616, 5832, 6036 and $6301 \mathrm{Da}$ and an absence of the $5583 \mathrm{Da}$ peak. This variation in the molecular masses of peptides is likely a result of the proteolytic processing of the precursor PI proteins to generate multiple functional PI species (equivalent to single IRD from multi-IRD CanPIs) [22,31,32].

The partially purified PI protein from uninduced and induced leaves, displaying varying mass spectral profiles on MALDI-TOF-MS, were separated on Tricine gel, and the proteins were individually excised and processed for in-gel digestion followed by the identification of peptides by MALDI-TOF-MS/MS (Additional file 1: Table S2). The $6 \mathrm{kDa}$ protein in all the extracts showed matches to 
A

\section{Cysteine variant $\square$ Reactive site variant}

Core reactive site

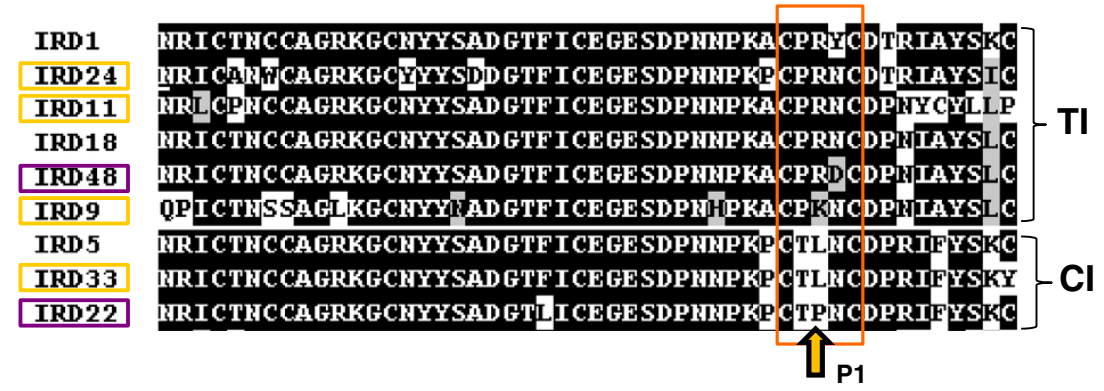

B

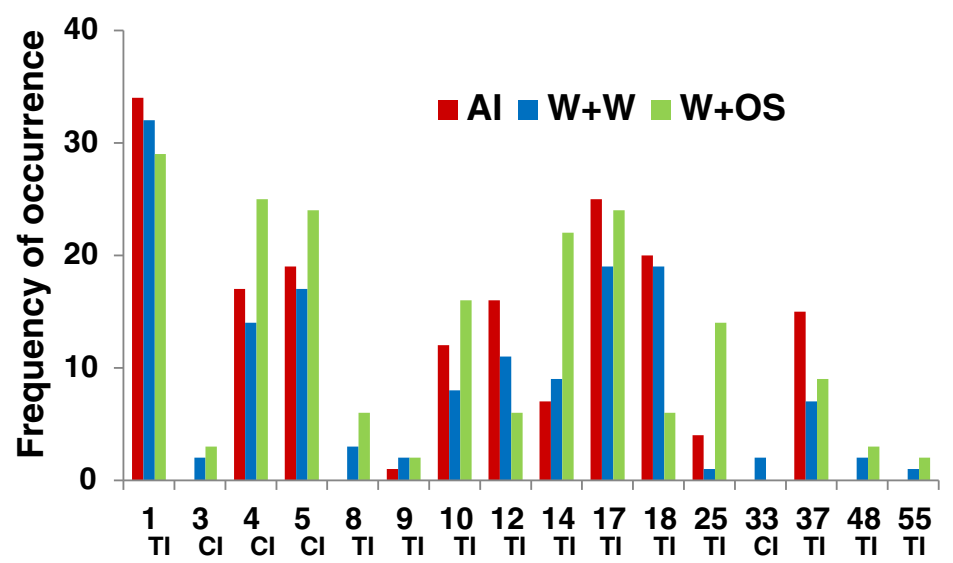

C

IRD number

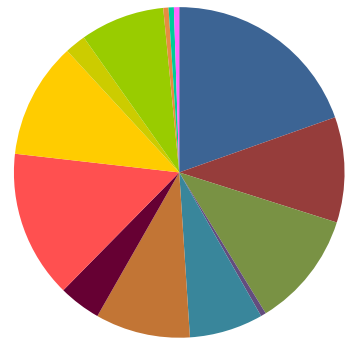

Al

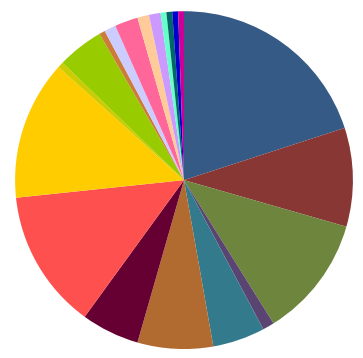

$\mathrm{W}+\mathrm{W}$

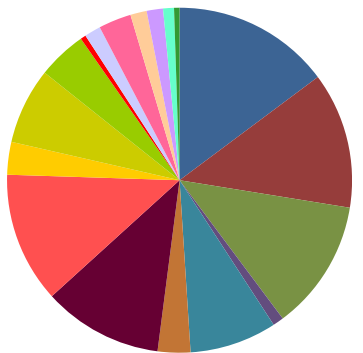

$\mathrm{W}+\mathrm{OS}$

Figure 3 Induction specific patterns of IRD expression. [A] Multiple sequence alignment of deduced aa sequences of selected IRD variants among all CanPI genes. IRD1/IRD18 represents a typical trypsin inhibitory domain (TI) and IRD5 represents a typical chymotrypsin inhibitory domain $(\mathrm{Cl})$. The selected IRDs showing variation in the number of cysteines or active site residues are marked by yellow and purple boxes, respectively. The reactive site residue P1 is marked by an arrow. The presence of Lys (K) or Arg (R) at the P1 site indicates trypsin inhibitory site, and Leu $(\mathrm{L})$ indicates chymotrypsin inhibitory site. The core reactive site is marked by an orange box. [B] The frequency of occurrence for individual IRDs per treatment. IRDs occurring only once were excluded from this graph. The highest representation of IRDs 1, 4, 5 and 17 across all treatments is apparent. [C] Pie charts represent the distribution of IRDs under each induction. A diverse array of unique IRDs is observed in wounded leaves treated with water or OS.

Pin-II proteinase inhibitors from C. annuum. However, due to high homology amongst IRD sequences, the database generated identification to several CanPIs/IRDs (Additional file 1: Table S2).
Tissue specificity of PI accumulation

Flowers, followed by stems and early fruit showed a significantly higher level of PI activity compared to tissue from leaves, roots and the different developmental 
A

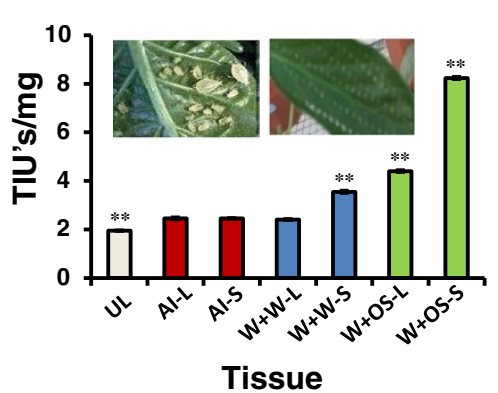

C

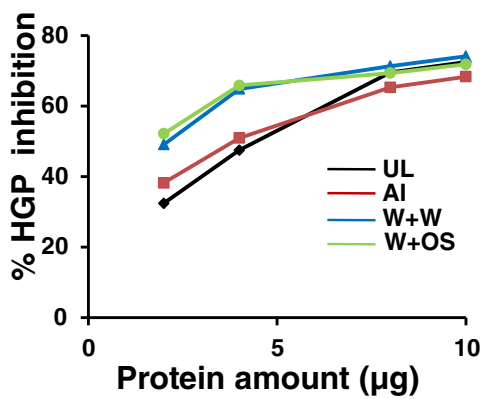

B

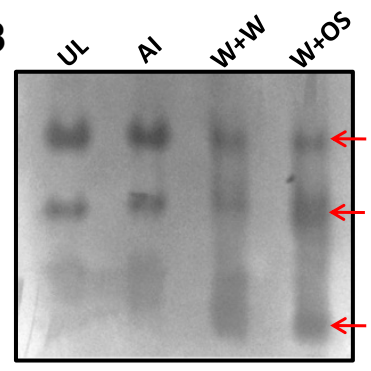

D

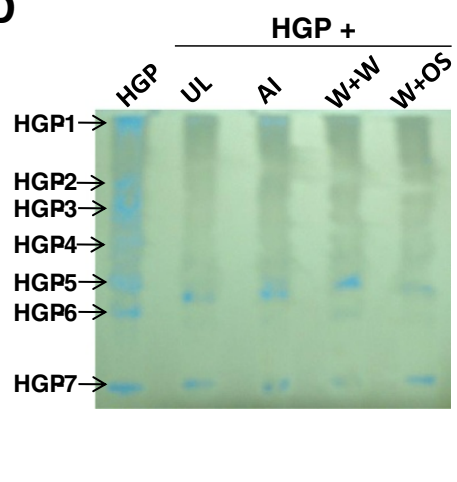

Figure 4 PI activity in C. annuum uninduced and induced leaves. [A] The accumulation of trypsin inhibitory activity in leaves infested with aphids (Al), wounded and treated with water $(W+W)$ or oral secretions (W+OS). Local (L) and systemic (S) tissues corresponding to the respective induction are represented. The W+OS systemic tissue shows statistically significant higher trypsin inhibitory units (TIUs) (Tukey's $t$-test at $p<0.01$ ). $[\mathbf{B}]^{*}$ In-gel TI profiles of induced C. annuum leaves. 3 TI bands were visualized in W+W and W+OS treated leaves while only 2 in uninduced and aphid infested leaves. [C] ${ }^{*}$ The percentage inhibition of HGP by Pls from leaf extracts estimated using azocasein as substrate. [D]* The inhibition of HGP isoforms by leaf extracts. Equal HGPI units of each of the extracts were incubated with HGP for $1 \mathrm{~h}$ at $2{ }^{\circ} \mathrm{C}$ and visualized for residual protease activity. ${ }^{*}$ The represented figures of induced tissues belong to systemic tissues of the particular induction treatment. Similar observations for local tissues of induction treatments.

stages of fruit (Additional file 4: Figure S3). Flower tissue showed the highest PI activity, while tissue from turning fruit exhibited the least activity with a 7-fold difference. The in-gel PI visualization after resolution on 2-DE for various $C$. annuum plant parts displayed the qualitative variations in the PI activity across these tissues (Figure 7). The clusters corresponding to 1-, 2-, 3- and 4-IRD CanPIs were prominent in stems, early fruit and flowers. In accordance to the very low TIUs in roots, we could detect only one TI isoform which corresponded to 2-IRD CanPI. The 1-IRD cluster contained several merged TI isoforms and indicated multiple, charge variants due to amino acid sequence differences. Stem tissue in particular showed less diversity in the 3-IRD cluster while exhibiting the highest number of charge variants in the 1-IRD cluster. Stem, early fruit stage and flower tissues revealed the presence of the high molecular mass isoforms of PIs (Figure 7, marked by arrows) that are predicted to be larger than those of 4-IRD CanPIs.

\section{Discussion}

C. annuum produces an array of PI genes exhibiting regulated expression under induced conditions. CanPIs are strongly elicited by wounding and upon Lepidopteran insect attack owing to the high cellular damage and plant's perception of herbivore-specific elicitors as compared to aphid infestation. Based on observed CanPI accumulation upon elicitation, it is intriguing to speculate that generating sequence diversity in the form of multi-IRD PIs is part of a plant's defense strategy to obtain a pool of diverse functional units to confine insect attack.

Among Solanaceae, different species of Nicotiana display PI genes containing 2 and 4 to 8 IRDs [19]. In addition, 2-IRD PIs from tomato (Solanum lycopersicum) and $S$. nigrum have been well characterized [4,33]. Simultaneously expressed PIs with varying IRD composition have been reported in $N$. alata stigma (4- and 6-IRDs; [34]), in N. glutinosa infected with TMV (6- and 8-IRD; [35]) and in $N$. attenuata in response to herbivory 


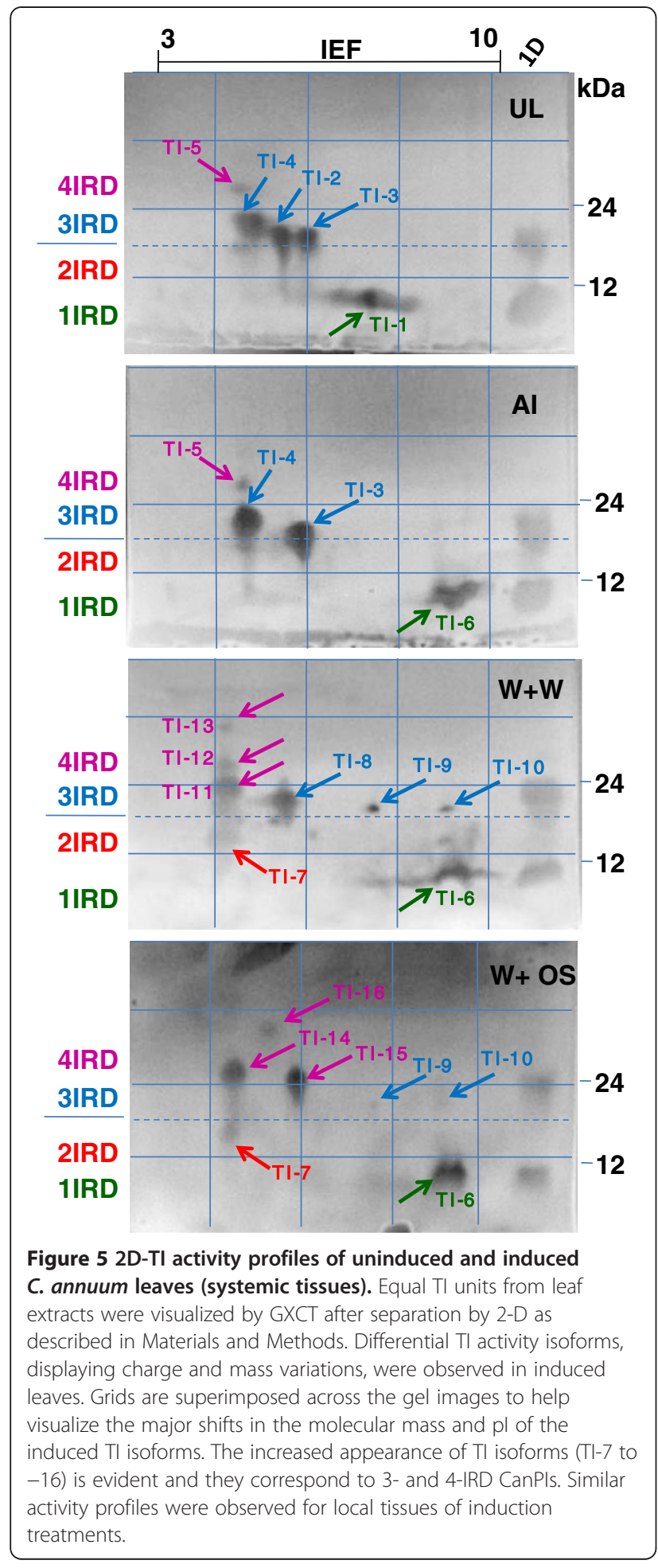

(7-IRD; [22]). In addition to the previously reported 23 PI genes from C. annuum pericarp, developing fruit and stem [16,26,27], 24 new CanPIs were isolated and characterized from the induced leaves in the present study. Among these 47 CanPI genes, 9 contained 4-IRDs, 20 contained 3-IRDs, 15 contained 2-IRDs and 3 contained a single IRD thus contributing to the diversity. Consistent with the previous studies, 3-IRD PIs were highly abundant in C. annuum leaves (Table 1, Figure 2). We observed a strong upregulation of 3- and 4-IRD CanPI transcripts in induced leaves when compared to uninduced leaves (Figure 1A, Table 1). Reciprocating patterns obtained at the PI activity level corroborate the induction specific regulation of CanPIs. Other reports on C. annuum have also demonstrated an increase in CanPI expression in local and systemic leaf tissues upon elicitation by aphids, viruses, insect feeding and mechanical wounding $[16,25,26]$. The stronger induction of PI activity in distant leaves than in local leaves challenged with the same treatment was interesting and suggested strong distant signalling.

Despite the difficulties of directly comparing the aphid infestation treatment with other treatments carried out in the present study, an interesting pattern of accumulated CanPIs is evident at transcript and PI activity level. CanPI transcript abundance in aphid infested leaves was much lower than that observed in wounded leaves treated with water or OS (Figure 1A). Aphids have been found to elicit defense related genes including PIs but the responses are low as compared to that elicited by chewing insect attack $[36,37]$. However, studies have also found aphid induced transcriptional signatures of salicylic acid signaling and no increase in PI transcripts [38]. In the present study, transcripts of CanPI-41 and -43 are highly accumulated under aphid infestation while CanPI-8 and -11 remain either un-elicited or suppressed by aphid damage; the latter being up-regulated by wounding or $\mathrm{W}+\mathrm{OS}$ treatment. The suppression of selected wound induced responses, rather than the lack of cellular damage, might also be responsible for the low responses to aphid attack [36]. Aphid infestation induced CanPI activity though much less as compared to wounded leaves treated with water or OS (Figure 4A). A unique aphid induced CanPI signature was evident in the $2 \mathrm{D}$ activity profiles (Figure 5 ).

Particularly, 4-IRD PIs were strongly induced in wounded leaves treated with OS (Figure 1B) highlighting the strong and specific effects of insect elicitors on CanPI regulation. Plant responses to wounding/insect feeding are known to be specifically altered by the plant's perception of herbivore-specific elicitors [8,39-42]. High amounts of jasmonic acid and the rapid accumulation of wound-inducible transcripts have been reported in response to insect damage or insect OS when compared to only mechanical wounding $[5,40,43]$. Wounded leaves treated with water showed the highest number (10) of uniquely expressed CanPIs (Figure 1C), though in lower frequency as compared to a few CanPIs $(-4,-7,-10)$ and 


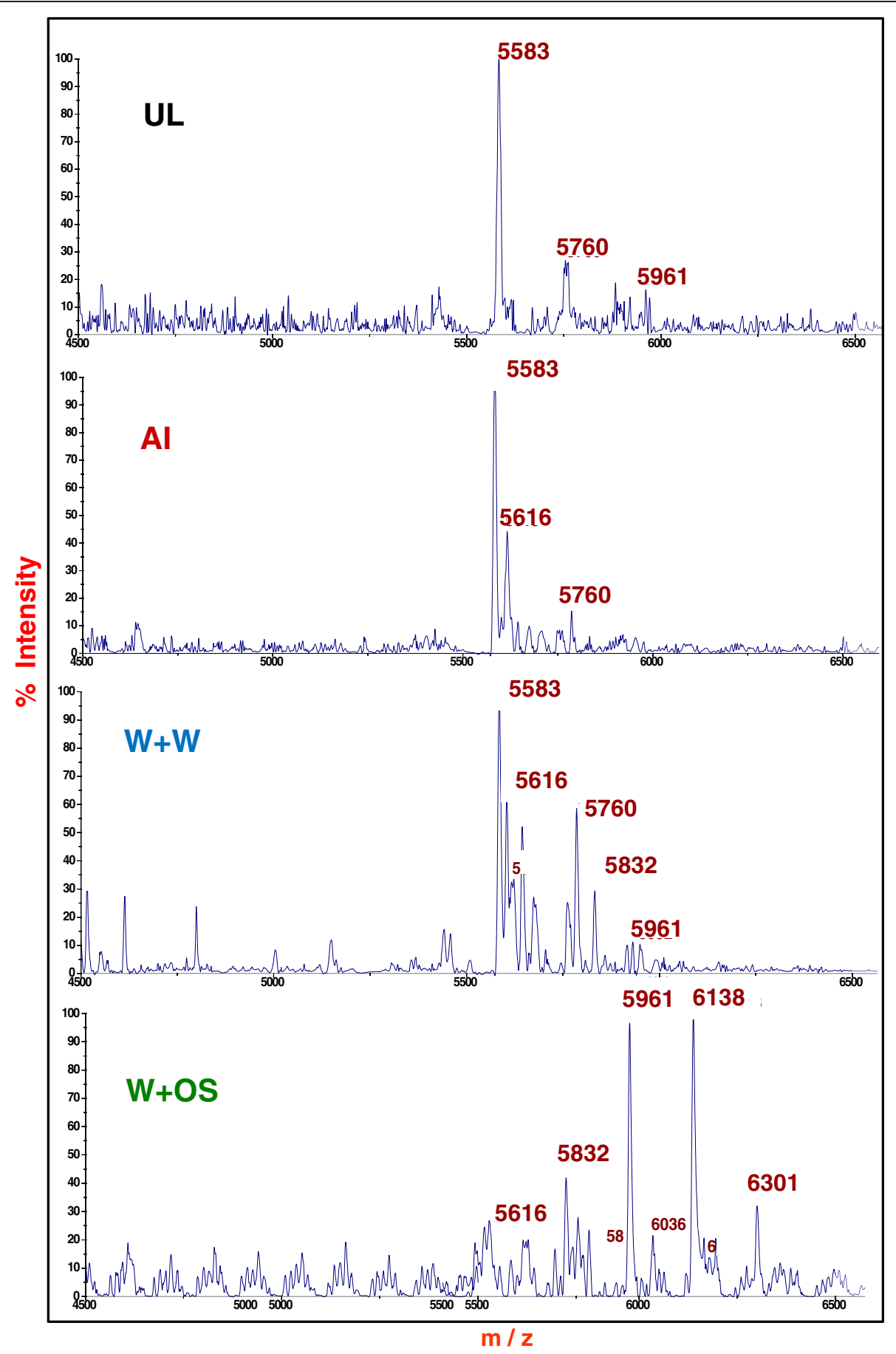

Figure 6 MALDI-TOF-MS characterization of partially purified PIs from leaf extracts (systemic tissue in case of inductions). Mass spectral analysis revealed peaks of varying masses in the range of 5.5 to $6.3 \mathrm{kDa}$ across treatments. These represent an 1-IRD peptide, which was further confirmed by MS/MS analysis. The increased diversity of processed IRDs is prominent in $C$. annuum leaf tissues wounded and treated with water $(\mathrm{W}+\mathrm{W})$ or oral secretions $(\mathrm{W}+\mathrm{OS})$ as compared to aphid infested.

IRDs $(4,5,10,14,25)$ with higher frequency in wounded leaves treated with OS. Specifically high representation of CanPIs with multiple IRDs directed towards enriching the PI blend with both CI and TI activities seems to be an approach adapted by the plant upon Lepidopteran insect attack, helpful in tackling a wide range of insect proteases [31]. Significantly high PI activity (Figure 4A) and detection of three PI activity bands in wounded leaves treated with water or OS in comparison to two activity bands in uninduced and aphid infested leaves 


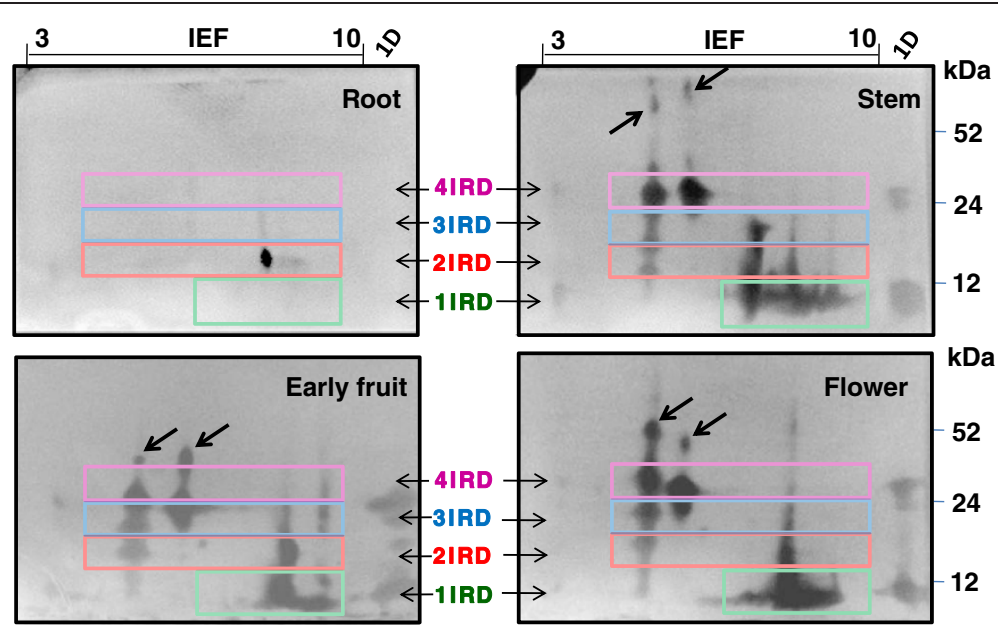

Figure 7 2D-TI activity profiles of various C. annuum plant parts under uninduced conditions. Several Tl activity isoforms were detected in all the tissues except root tissue, which shows very low TI activity and only one TI isoform. Qualitative and quantitative variations in TI profiles of tissue from stem, early fruit and flower are quite evident and the differences are highlighted through the clusters of 1-, 2-, 3- and 4-IRD PIs.

(Figure 4B) is indicative of quantitative and qualitative variations in the accumulated PI activity. Further characterization by $2 \mathrm{D}$ electrophoresis, revealed the presence of multiple charge and/or molecular mass variants observed in wounded leaves treated with water (Figure 5; TI-7 to -13) and with OS (Figure 5; TI-14 to TI-16) clearly indicating the induced isoform diversity. The differential isoforms detected mostly corresponded to 3- and 4-IRD PIs and thus correlates with the high CanPI transcript accumulation under these two treatments. The absence of certain TI isoforms in OS treated leaves compared to wounding alone, suggests the suppression of some induced responses, resulting in treatment specific patterns. With respect to HGP inhibition potential, the PI activity in all leaf tissues attained 70\% inhibition of HGP (Figure 4C) and could inhibit almost all the HGP isoforms (Figure 4D). However, an early saturation of HGP inhibition by proteins from leaves induced by wounding and/or treated with OS, as compared to uninduced leaves, is suggestive of the high quantitative accumulation of PI units in such leaf tissues and higher specific activity against insect gut proteases. Multiple IRDs are known to be generated from precursor $N$. alata PI proteins in Me-JA-elicited leaves [22,44] and from CanPI precursor proteins by the action of endogenous proteases at the linker regions [16]. An increase in the number and intensity of variant mass peaks equivalent to single IRDs, in wounding with water and with OS protein fractions (Figure 6), suggests the enhanced proteolytic processing of the up-regulated CanPI precursor proteins [22,31,32]. The specific presence of isoform TI-6 and absence of TI-1 (Figure 5) under all inductions also indicate the accumulation of differential 1-IRD isoforms that are generated as a result of processing of CanPI precursors. Thus, our results substantiate the hypothesis [22] that elicitation leads to over-production of the CanPI precursors and enhanced, differential processing of the precursors by proteases to IRDs, resulting in structurally and functionally diverse processed products. It was also noticed that induction treatment specificity is maintained even at the level at which precursor proteins are processed. Peaks ranging from 5.9 to $6.3 \mathrm{kDa}$ show high intensity in wounding with OS treatment whereas the peaks from 5.5 to 5.8 $\mathrm{kDa}$ are prominent in uninduced and other treatments, aphid infested and wounding treated with water. These results affirm that plants can differentially perceive various kinds of biotic stresses and respond appropriately through regulation of PIs at transcriptional and posttranslational levels.

Sequence analysis revealed highly homologous CanPIs with an average variance of $4 \%$. The clear absence of partial N- and C-terminal repeats in the CanPI precursors groups them as distinct from $N$. benthamiana PinII PIs (Figure 2). The explicit clustering of C. annuum PIs from all other Solanaceous Pin-II PIs suggests recent evolutionary origins [21]. The diversity in CanPIs can be attributed to individual IRDs, which display a sequence variation ranging from 2 to $20 \%$ within the vicinity of the reactive site loops and C-terminal region. Twenty eight unique IRDs, constituting 7 CIs and 21 TIs, follow the H-L type topology, where the sequence repeat is identical to the structural repeat [21]. The induction-specific IRD distribution is predominantly biased towards TIs rather than CIs. It is known that in Lepidopteran insects, trypsin-like proteases are predominant which could be correlated to the relatively high abundance of trypsin specific PIs in plants. 
Active site variants of TIs 'CPRNC, 'CPKNC, 'CPRYC' and 'CPRDC' and two types of CI sites 'CTLNC' and 'CTPNC' were present among all identified 28 IRDs. Interestingly four cysteine variants either, missing one or more conserved cysteine residues, change in position of cysteines or having additional cysteines were identified in the present study (Figure 3A). Recently, six natural IRD variants with selective losses of cysteine residues have been identified in potato [45]. The loss of cysteine residues is often associated with functional differentiation and suggests positive evolutionary gene selection. However, the nature of mutations and the associated factors responsible for selective losses of cysteines remains unclear. Studies on the significance of such mutations affecting the proteinase binding affinity and structural stability/integrity of IRDs have initiated an active debate on the evolution of disulfide bonds in the Pin-II family $([45,46]$, our unpublished results). Particularly in C. annuum, the identification of four such IRDs as a result of various inductions suggests that plants elaborative defenses by expressing modified IRDs that improve their overall activity against target proteases. Among various tissues from C. annuum, flowers revealed the highest accumulation of PI activity (Additional file 4: Figure S3), consistent with a role in protecting the reproductive parts of the plant against pests as reported in tomato $[15,44]$.

\section{Conclusions}

This study suggests that CanPI sequence diversity, tissue specificity and explicit responses to different inductions are part of effective plant defense system. The significance of huge complexity of PIs observed specifically in C. annuum needs to be understood. Recent reports on the endogenous and/or defensive roles of PIs from various Solanaceous species and simultaneous expression of multiple CanPIs constitutively highlight their prospective involvement in many of the plant's complex processes [16,19,23,47-49]. Moreover, up-regulated yet specialized CanPI expression upon wounding and insect infestation provides insights into the evolution of PI based plant defense mechanisms against insects and generates many unanswered questions about their regulation. Essentially, more functional studies need to be performed for specific CanPI genes in order to ascertain their roles under a particular treatment and how this variation accounts for the fitness benefit of the plant under specific biotic stress conditions.

\section{Methods}

Plant material and induction treatments

C. annuum seeds (cv PhuleJyoti) (diploid) were grown in pots with Soilrite (Mixture of horticulture grade expanded perlite, Irish Peat moss and exfoliated vermiculite in equal ratio; Naik Krushi, Pune, MS, India) and supplemented daily with Hoagland solution. 30-dayold seedlings were transferred to individual pots and grown in a growth chamber maintained at $23^{\circ} \mathrm{C}\left( \pm 2^{\circ} \mathrm{C}\right)$ with a $14 \mathrm{~h}$ light photoperiod. Leaves, stems, various stages of fruits (early, mid, turning and late), roots and flowers from mature plants (3 months old) were harvested for screening tissue-specific CanPIs.

All induction experiments were performed on 3month-old plants and a set of 3 plants was taken per treatment for each biological replicate. Leaves were mechanically wounded by rolling a fabric pattern wheel along the length of the lamina (4 to 6 rolls depending on the size of leaf) and the resulting puncture wounds were immediately treated with water or OS. These were considered local tissue, whereas the non-wounded leaves one node above or below were harvested to measure systemic responses. Local and systemic tissues were collected after $30 \mathrm{~h}$ of treatment. OS used was collected from $H$. armigera larvae and diluted 1:50 (v/v) times in MQ water. Plants were kept in an open garden for passive aphid infestation. Natural infestation by Myzus persicae was observed on $C$. annuum leaves within a week. The density of aphids was high on the leaf lamina towards the petiole. Leaves with at least 20 nymphs per leaf growing at the same nodes were collected as local tissue whereas non-infested leaves were harvested as systemic tissues. Since the plants were naturally infested by aphids in open conditions, the possible comparisons with this treatment and the other two, which were performed in a highly specific and controlled manner, are limited. Leaves from un-elicited control plants growing at the same nodes were harvested from uninduced plants. All the tissue collections were done at a same time and were flash-frozen in liquid nitrogen and stored at $-80^{\circ} \mathrm{C}$ until further use. Two biological replicates were used for the whole study.

\section{Expression profiling, cloning of Pin-II genes and sequence analysis}

Total RNA from C. annuum leaf tissues (uninduced and all three inductions-systemic) was isolated using TRIZOL (Invitrogen, Carlsbad, CA, USA) followed by DNAse treatment at $37^{\circ} \mathrm{C}$. Purified RNA was quantified by spectrophotometry, and $1.5 \mu \mathrm{g}$ was used for firststrand cDNA synthesis using a reverse transcriptase kit (Promega, Madison, WI, USA). Proof-reading Accuprime Pfx DNA polymerase (Invitrogen) was used to amplify cDNAs from systemic leaf tissues of individual treatments and uninduced leaf in independent PCR reactions using CanPI gene (Genbank accession: AF039398) specific primer pair (CanPin-1F and CanPin-1R; Additional file 1 Table S1A). Amplicons were cloned into pGEMTeasy vector (Promega). 60 cloned fragments from each treatment were sequenced using standard T7 forward 
and SP6 reverse primers. Sequence editing and analysis was carried out using BioEdit, Clustal-X and Lasergene software. For semi-quantitative analysis of CanPIs within the tissues, independent PCRs were performed with reduced number of cycles (25), Accuprime Pfx DNA polymerase and CanPI gene specific primer pair. Specific primer pairs were designed for individual CanPI genes in order to check the expression of specific CanPIs. However, due to a high degree of similarity/homology within C. annuum PIs, it was possible to design genespecific primer pairs for CanPI-3, $-5,-7,-8$ and -10 only. The specific pairs of oligonucleotides used for the internal differentiation of individual CanPIs are stated in Additional file 1: Table S1A and B. All PCRs were carried out in technical duplicates.

Protein extraction and proteinase inhibitor activity assays Total soluble protein was extracted from $1 \mathrm{~g}$ of fresh leaf tissue obtained from uninduced and induced (local and systemic for all treatments) C. annuum, using a mixture of water and $5 \%$ polyvinylpolypyrrolidone (Sigma, St. Louis, MO, USA). Following protein estimation using Bradford reagent (Bio-Rad Laboratories, Hercules, CA, USA), the trypsin inhibitory activity and $H$. armigera gut protease (HGP) inhibitory activity was estimated enzymatically in the leaf extracts using the synthetic substrate Benzoyl-DL-Arginyl- $p$-Nitroanilide (Sigma, St. Louis, MO, USA) as described earlier [28]. Protease inhibitor activity was expressed as trypsin inhibitory units per mg of tissue (TIUs/mg). Various amounts of the leaf protein extracts were titrated against HGP to determine maximum inhibitory activity. All the assays were carried out in technical triplicates with 2 biological replicates and statistical analysis of data was performed using singlefactor ANOVA followed by Tukey's post hoc analysis.

\section{D and 2D electrophoresis for in-gel identification of proteinase inhibitory activity}

Equal TIUs of leaf protein extracts were resolved on $12 \%$ native-PAGE and further processed in order to visualize trypsin inhibitor isoforms using the previously described gel X-ray film contact print (GXCT) method [50]. To visualize protease activity, protein extracts incubated with HGP were resolved on $8 \%$ native-PAGE gel and processed by GXCT [51]. For 2D electrophoresis, acetone-precipitated proteins were re-suspended in rehydration buffer and separated in first dimension (Isoelectric focusing) using 11-cm IPG strips (pH 3-10 NL; Bio-Rad Laboratories, Hercules, CA, USA) as per the manufacturer's protocol. Second-dimension separation was done on $12 \%$ SDS-PAGE gel using a Hoefer electrophoresis unit (GE Healthcare Bio-sciences AB, Buckinghamshire, UK) maintained at $24^{\circ} \mathrm{C}$ and $200 \mathrm{~V}$. Trypsin inhibitor activity was visualized by modified GXCT [52].
All the electrophoresis experiments were carried out in technical triplicates with 2 biological replicates.

\section{Partial purification and proteomic analysis of leaf protein extracts}

To identify the proteinase inhibitors from C. annuum leaf tissues, protein extracts were first partially purified in the following manner. Ammonium-sulphate-precipitated proteins ( $90 \%$ saturation) from leaf extracts were resuspended in $50 \mathrm{mM}$ Tris buffer ( $\mathrm{pH} 8.0)$, and subsequently treated at $65^{\circ} \mathrm{C}$ for $10 \mathrm{~min}$ before being desalted using PD SpinTrap ${ }^{\mathrm{TM}}$ G-25 column (GE Healthcare). Proteins were then separated on DEAE-Sephacel (GE Healthcare) equilibrated

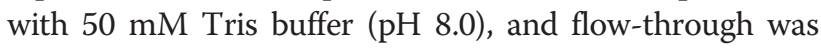
collected separately from bound proteins that were eluted with a $\mathrm{NaCl}$ gradient of $0.25 \mathrm{M}$ to $0.4 \mathrm{M}$ in $50 \mathrm{mM}$ Tris buffer ( $\mathrm{pH}$ 8.0). Fractions with trypsin inhibitor activity were concentrated and desalted using a PD Spintrap column. The partially purified protein fractions were pooled and qualitatively analyzed by matrix-assisted laser desorption ionization time-of-flight mass spectrometry (MALDI-TOF-MS), Voyager-De-STR (Applied Biosystems, Framingham, MA, USA). Mass spectral acquisition was performed with a standard instrumental protocol as described earlier [31]. In brief, $2 \mu \mathrm{g}$ of protein sample was mixed with $20 \mu \mathrm{L}$ of freshly prepared sinapinic acid (Sigma-Aldrich) (in 30\% acetonitrile [ACN], 0.1\% trifluoroacetic acid [TFA]) and spotted on the stainless steel MALDI plate, and spectral profiles were acquired in the range of 1 to $25 \mathrm{kDa}$. The spectra were analyzed with Data Explorer ${ }^{\mathrm{TM}}$ for regions of interest and processed for advanced base-line correction and noise removal.

To obtain protein sequence data, the partially purified proteins were separated on $16 \%$ Tricine SDS-PAGE gel [53] to resolve the low molecular mass proteins, after which protein bands were in-gel digested and analyzed by MALDI-Q-TOF- MS (SYNAPT High Definition Mass spectrometer, Waters Corporation, Milford, MA, USA). Mass spectral acquisition was carried out by MALDI survey method. Protein Lynx Global server version 2.4 software (Waters) was used for data processing and database searches. The MS/MS data was searched against the Pin-II protein database constructed separately using the following parameters: peptide tolerance of $20 \mathrm{ppm}$, fragment tolerance of $0.05 \mathrm{D}$, one missed cleavage, carbamidomethylation of cysteines and possible oxidation of Methionine.

\section{Additional files}

Additional file 1: Table S1. Oligonucleotide primers used for RT-PCR and CanPl internal differentiation. Table S2: Protein identification by MALDI-TOF-MS/MS, database searches. 
Additional file 2: Figure S1. Multiple sequence alignment of deduced aa sequences of signal peptides (SP-1 to SP-10) of CanPI genes, displaying variations.

Additional file 3: Figure S2. Multiple sequence alignment of deduced aa sequences of IRDs (28 in number) constituting all the CanPI genes. The reactive site residue $P 1$ is marked by an arrow. Presence of $L y s(K)$ or $\operatorname{Arg}(R)$ at $P 1$ site, indicates trypsin inhibitory site $(T I)$ and Leu $(L)$ indicates chymotrypsin inhibitory site $(\mathrm{Cl})$. The core reactive site is marked by an orange box.

Additional file 4: Figure S3. Tissue-specific Tl activity in various tissues of a mature $\mathrm{C}$. annuum plant. Concentration is represented in terms of trypsin inhibitory units (TIUs/mg). Flower tissue shows the highest TIUs with an almost 7-fold increase compared to leaf tissue. Stem and early fruit tissue also shows significantly higher $\mathrm{Tl}$ activity.

\section{Abbreviations}

Aa: Amino acid; Cl: Chymotrypsin inhibition; HGPI: H. armigera gut protease inhibition; MALDI-TOF-MS: Matrix-assisted laser desorption ionization time-offlight mass spectrometry; IRDs: Inhibitory repeat domains; OS: Oral secretions; PI: Proteinase inhibitor; TI: Trypsin inhibition.

\section{Competing interests}

The authors declare that they have no competing interests.

\section{Authors' contribution}

MM carried out the induction experiments, sequence analysis, characterization of PI protein activity and drafted the manuscript. NSM was involved in protein extractions and the 2D gel electrophoresis. VAT participated in design of the study, sequencing data analysis and helped to draft the manuscript. MJK helped in performing the MALDI-TOF-MS analysis and protein identifications. ITB and VSG helped in interpretation of results and drafting the final version of the manuscript. APG conceived the study and helped in drafting the manuscript. All authors contributed at the draft stage of writing and all approved the final manuscript.

\section{Acknowledgments}

The Council of Scientific and Industrial Research (CSIR), Government of India, New Delhi supported this project under network project grants to National Chemical Laboratory, Pune (NWP0003). MM, NSM and VAT acknowledge CSIR for research fellowships. Authors acknowledge S. S. Deo from NCL, Pune for help in mass spectrometry, Dr. Klaus Gase, MPI-CE, Jena; Dr. N. Y. Kadoo and Rasika Bhagwat, NCL, Pune for DNA sequencing. The critical comments of Dr. Dhanasekaran Shanmugam on the manuscript were enormously helpful and we thank Emily Wheeler for editorial assistance. The authors have declared no conflict of interest.

\section{Author details}

${ }^{1}$ Plant Molecular Biology Unit, Division of Biochemical Sciences, CSIR-National Chemical Laboratory, Dr. Homi Bhabha Road, Pune, MS 411 008, India. ${ }^{2}$ Department of Molecular Ecology, Max Planck Institute for Chemical Ecology, Jena 07745, Germany. ${ }^{3}$ Present address: Institute of Bioinformatics and Biotechnology, University of Pune, Pune, MS 411 007, India.

Received: 6 April 2012 Accepted: 7 November 2012

Published: 16 November 2012

\section{References}

1. Karban R, Baldwin IT: Induced responses to herbivory. Chicago: University of Chicago Press; 1997:1-319.

2. Haruta M, Major IT, Christopher ME, Patton JJ, Constabel CP: A Kunitz trypsin inhibitor gene family from trembling aspen (Populus tremuloides Michx.): cloning, functional expression, and induction by wounding and herbivory. Plant Mol Biol 2001, 46:347-359.

3. Van Dam NM, Witjes L, Svatos A: Interactions between aboveground and belowground induction of glucosinolates in two wild Brassica species. New Phytol 2004, 161:801-810.

4. Hartl M, Giri AP, Kaur H, Baldwin IT: Serine protease inhibitors specifically defend Solanum nigrum against generalist herbivores but do not influence plant growth and development. Plant Cell 2010, 22:4158-4175.
5. Walling $L L$ : The myriad plant responses to herbivores. Journal of Plant Growth Regul 2000, 19:195-216.

6. Baldwin IT, Halitschke R, Kessler A, Schittko U: Merging molecular and ecological approaches in plant-insect interactions. Curr Opinion in Plant Biol 2001, 4:351-358

7. Jianqiang $W$, Baldwin IT: New insights into plant responses to the attack from insect herbivores. Annu Rev Genet 2010, 44:1-24.

8. Hermsmeier D, Schittko U, Baldwin IT: Molecular interactions between the specialist herbivore Manduca sexta (Lepidoptera, Sphingidae) and its natural host Nicotiana attenuata. I. Large-scale changes in the accumulation of growth- and defense-related plant mRNAs. Plant Physiol 2001, 125:683-700.

9. Giri AP, Wunsche H, Mitra S, Zavala JA, Muck A, Svatos A, Baldwin IT: Molecular interactions between the specialist herbivore Manduca sexta (Lepidoptera, Sphingidae) and its natural host Nicotiana attenuata. VII. changes in the plant's proteome. Plant Physiol 2006, 142:1621-1641.

10. Green TR, Ryan CA: Wound-induced proteinase inhibitor in plant leaves: A possible defense mechanism against insects. Science 1972, 175:776-777.

11. Bryant J, Gurusaddaiah T, Ryan CA: Proteinase Inhibitor I1 from potatoes: Isolation and characterization of its promoter components. Biochemistry 1976, 15:3418-3423.

12. Plunkett G, Senear DF, Zuroske G, Ryan CA: Proteinase inhibitors I and II from leaves of wounded tomato plants: Purification and properties. Arch Biochem Biophys 1982, 213:463-472.

13. Ryan CA: Protease inhibitors in plants: Genes for improving defenses against insects and pathogens. Ann Review Phytopathol 1990, 28:425-449.

14. Ryan CA: The systemin signaling pathway: Differential activation of plant defensive genes. Biochim Biophys Acta 2000, 1477:112-121.

15. Damle MS, Giri AP, Sainani MN, Gupta VS: Higher accumulation of proteinase inhibitors in flowers than leaves and fruits as a possible basis for differential feeding preference of Helicoverpa armigera on tomato (Lycopersicon esculentum Mill, cv. Dhanashree). Phytochemistry 2005, 66:2659-2667.

16. Tamhane VA, Giri AP, Kumar P, Gupta VS: Spatial and temporal expression patterns of diverse Pin-II proteinase inhibitor genes in Capsicum annuum Linn. Gene 2009, 442:88-98.

17. Garcia-Olmedo F, Salcedo G, Sánchez-Monge RF, Gomez L, Royo J, Carbonero P: Plant proteinaceous inhibitors of proteinases and aamylases. Oxford Surveys of Plant Mol and Cell Biol 1987, 4:275-334.

18. Patankar AG, Harsulkar AM, Giri AP, Gupta VS, Sainani MN, Ranjekar PK, Deshpande W: Diversity in inhibitors of trypsin and Helicoverpa armigera gut proteinases in chickpea (Cicer arietinum) and its wild relatives. Theor Appl Genet 1999, 99:719-726.

19. Wu J, Hettenhausen C, Baldwin IT: Evolution of proteinase inhibitor defenses in North American allopolyploid species of Nicotiana. Planta 2006, 224:750-760.

20. Barta E, Pintar A, Pongor S: Repeats with variations: accelerated evolution of the Pin2 family of proteinase inhibitors. Trends Genet 2002, 18:600-603.

21. Kong $L$, Ranganathan S: Tandem duplication, circular permutation, molecular adaptation: How Solanaceae resists pests via inhibitors. BMC Bioinformatics 2008, 9(Suppl 1):S22.

22. Horn M, Patankar AG, Zavala JA, Wu J, Doleckova-Maresova L, Vujtechova M, Mares M, Baldwin IT: Differential elicitation of two processing proteases controls the processing pattern of the trypsin proteinase inhibitor precursor in Nicotiana attenuata. Plant Physiol 2005, 139:375-388.

23. Hartl M, Giri AP, Kaur H, Baldwin IT: The multiple functions of plant serine protease inhibitors: Defense against herbivores and beyond. Plant Signalling and Behaviour 2011, 6:1-3.

24. Antcheva N, Pintar A, Patthy A, Simoncsits A, Barta E, Tchorbanov B, Pongor S: Proteins of circularly permuted sequence present within the same organism: the major serine proteinase inhibitor from Capsicum annuum seeds. Protein Sci 2001, 10:2280-2290.

25. Moura DS, Ryan CA: Wound-inducible proteinase inhibitors in pepper. Differential regulation upon wounding, systemin, and methyl jasmonate. Plant Physiol 2001, 126:289-298

26. Kim S, Hong Y-N, An CS, Lee K-W: Expression characteristics of serine proteinase inhibitor II under variable environmental stresses in hot pepper (Capsicum annuum L.). Plant Sci 2001, 161:27-33.

27. Shin R, Lee GJ, Park CJ, Kim TY, You JS, Nam YW, Paek KH: Isolation of pepper mRNAs differentially expressed during the hypersensitive 
response to tobacco mosaic virus and characterization of a proteinase inhibitor gene. Plant Sci 2001, 161:727-737.

28. Tamhane VA, Chougule NP, Giri AP, Dixit AR, Sainani MN, Gupta VS: In vivo and in vitro effect of Capsicum annum proteinase inhibitors on Helicoverpa armigera gut proteinases. Biochim Biophys Acta 2005, 1722:156-167.

29. Tamhane VA, Giri AP, Sainani MN, Gupta VS: Diverse forms of Pin-II family proteinase inhibitors from Capsicum annuum adversely affect the growth and development of Helicoverpa armigera. Gene 2007, 403:29-38.

30. Boden M, Hawkins J: Prediction of subcellular localisation using sequence-biased recurrent networks. Bioinformatics 2005, 21:2279-2286.

31. Mishra M, Tamhane VA, Khandelwal N, Kulkarni MJ, Gupta VS, Giri AP: Interaction of recombinant CanPIs with Helicoverpa armigera gut proteases reveals their processing patterns, stability and efficiency. Proteomics 2010, 10:2845-2857.

32. Huesgen $\mathrm{P}$, Fand Overall CM: N- and C-terminal degradomics: new approaches to reveal biological roles for plant proteases from substrate identification. Physiol Plantarum 2012, 145(1):5-17.

33. Graham JS, Pearce G, Merryweather J, Titani K, Ericsson LH, Ryan CA: Wound-induced proteinase inhibitors from tomato leaves. II. The cDNAdeduced primary structure of pre-inhibitor II. Journal of Biol Chem 1985, 260:6561-6564.

34. Miller EA, Lee MC, Atkinson AH, Anderson MA: Identification of a novel four-domain member of the proteinase inhibitor II family from the stigmas of Nicotiana alata. Plant Mol Biol 2000, 42:329-333.

35. Choi D, Park JA, Seo YS, Chun YJ, Kim WT: Structure and stress-related expression of two cDNAs encoding proteinase inhibitor II of Nicotiana glutinosa L. Biochim Biophys Acta 2000, 1492:211-215.

36. Voelckel C, Weisser WW, Baldwin IT: An analysis of plant-aphid interactions by different microarray hybridization strategies. Mol Ecology 2004, 13:3187-3195

37. Zhu-Salzman K, Salzman RA, Ahn JE, Koiwa H: Transcriptional regulation of sorghum defense determinants against a phloem-feeding aphid. Plant Physiol 2004, 134:420-431.

38. Fidantsef AL, Stout MJ, Thaler JS, Duffey SS, Bostock RM: Signal interactions in pathogen and insect attack: expression of lipoxygenase, proteinase inhibitor II, and pathogenesis-related protein P4 in the tomato, Lycopersicon esculentum. Physiol Mol Plant Pathol 1999, 54:97-114.

39. Thaler JS, Bostock RM: Interactions between abscisic-acid-mediated responses and plant resistance to pathogens and insects. Ecology 2004, 85:48-58.

40. Reymond $\mathrm{P}$, Weber $\mathrm{H}$, Damond M, Farmer EE: Differential gene expression in response to mechanical wounding and insect feeding in Arabidopsis. Plant Cell 2000, 12:707-719.

41. Diezel C, Kessler D, Baldwin IT: Pithy protection: Nicotiana attenuata's jasmonic acid-mediated defenses are required to resist stem-boring weevil larvae. Plant Physiol 2011, 155:1936-1946.

42. Voelckel C, Schittko U, Baldwin IT: Herbivore-induced ethylene burst reduces fitness costs of jasmonate- and oral secretion induced defenses in Nicotiana attenuata. Oecologia 2001, 127:274-280.

43. Korth KL, Dixon RA: Differential transcript accumulation near wound sites in response to insect or mechanical damage. Plant Physiol 1997, 11:4-26.

44. Atkinson AH, Heath RL, Simpson RJ, Clarke AE, Anderson MA: Proteinase inhibitors in Nicotiana alata stigmas are derived from a precursor protein which is processed into five homologous inhibitors. Plant Cell 1993, 5:203-213.

45. Li X-Q, Zhang T, Donnelly D: Selective loss of cysteine residues and disulphide bonds in a potato proteinase inhibitor II family. PLOS One 2011, 6:e18615.

46. Schirra HJ, Guarino RF, Anderson MA, Craik DJ: Selective removal of individual disulfide bonds within a potato type II serine proteinase inhibitor from Nicotiana alata reveals differential stabilization of the reactive-site loop. Journal of Mol Biol 2010, 395:609-626.

47. Johnson ED, Miller EA, Anderson MA: Dual location of a family of proteinase inhibitors within the stigmas of Nicotiana alata. Planta 2007, 225:1265-1276.

48. Sin SF, Chye ML: Expression of proteinase inhibitor II proteins during floral development in Solanum americanum. Planta 2004, 219:1010-1022.

49. Xu ZF, Teng WL, Chye ML: Inhibition of endogenous trypsin- and chymotrypsin-like activities in transgenic lettuce expressing heterogeneous proteinase inhibitor SaPIN2a. Planta 2004, 218:623-629.
50. Pichare MM, Kachole MS: Detection of electrophoretically separated protease inhibitors using X-ray film. J Biochem Biophys Methods 1994, 28:215-224.

51. Harsulkar AM, Giri AP, Patankar AG, Gupta VS, Sainani MN, Ranjekar PK, Deshpande W: Successive use of non-host plant proteinase inhibitors required for effective inhibition of Helicoverpa armigera gut proteinases and larval growth. Plant Physiol 1999, 121:497-506.

52. Giri AP, Harsulkar AM, Ku MSB, Gupta VS, Deshpande W, Ranjekar PK, Franceschia VR: Identification of potent inhibitors of Helicoverpa armigera gut proteinases from winged bean seeds. Phytochemistry 2003, 63:523-532.

53. Schägger H: Tricine-SDS-PAGE. Nat Protoc 2006, 1:16-22.

doi:10.1186/1471-2229-12-217

Cite this article as: Mishra et al:: Stress inducible proteinase inhibitor diversity in Capsicum annuum. BMC Plant Biology 2012 12:217.

\section{Submit your next manuscript to BioMed Central and take full advantage of:}

- Convenient online submission

- Thorough peer review

- No space constraints or color figure charges

- Immediate publication on acceptance

- Inclusion in PubMed, CAS, Scopus and Google Scholar

- Research which is freely available for redistribution

Submit your manuscript at www.biomedcentral.com/submit
C) Biomed Central 